\title{
The strong law of large numbers for sums of randomly chosen random variables
}

\author{
Agnieszka M. Gdula and Andrzej Krajka \\ Maria-Curie Skłodowska University, ul. Akademicka 9, 20-033 Lublin, Poland \\ (e-mail: gdula.agnieszka@gmail.com; akrajka@gmail.com)
}

Received January 30, 2020; revised October 16, 2020

\begin{abstract}
Let $\left\{X_{n}, n \geqslant 1\right\}$ be a sequence of independent or identically distributed dependent random variables, and let $\left\{A_{n}, n \geqslant 1\right\}$ be a sequence of random subsets of natural numbers independent of $\left\{X_{n}, n \geqslant 1\right\}$. In this paper, we describe the strong law of large numbers (SLLN) of the form $\sum_{i \in A_{n}}\left(X_{i}-\mathbf{E} \sum_{i \in A_{n}} X_{i}\right) / b_{n} \rightarrow 0$ a.s. as $n \rightarrow \infty$ for some sequence of nondecreasing positive numbers $\left\{b_{n}, n \geqslant 1\right\}$. There often arises an assumption that $\left\{A_{n}, n \geqslant 1\right\}$ are almost surely increasing: $A_{n} \subset A_{n+1}$, a.s. $n \geqslant 1$.
\end{abstract}

MSC: 60F15

Keywords: strong law of large numbers, randomly indexed sums, random sets

\section{Introduction}

Let $\left\{X_{n}, n \geqslant 1\right\}$ be a sequence of independent random variables defined on a probability space $(\Omega, \mathfrak{F}, \mathbf{P})$ such that $\mathbf{E}\left|X_{n}\right|<\infty, n \geqslant 1$. A random set with values in $2^{\mathbb{N}}$ is a map $A: \Omega \rightarrow 2^{\mathbb{N}}$ such that $A^{-1}(B)=$ $\{\omega \in \Omega: A(\omega)=B\} \in \mathfrak{F}$ for any $B \subset \mathbb{N}$ (cf. [7, p. 35, Def. 3.1 and the remark on p. 72]). Obviously, if $A$ and $B$ are random sets, then $A \cup B, A \cap B$, and $A \backslash B$ are also random sets. Furthermore, $|A|$ is a nonnegative random variable, where $|A|$ is the cardinality of the set $A$. The basis of the theory of random sets can be found in a classic book by Matheron [5] or, more currently, in Molchanov's book [6] or Nguyen's book [7]. For an arbitrary (random or nonrandom) subset $A$ of natural numbers, we put

$$
S(A)=\sum_{i \in A} X_{i}-\mathbf{E} \sum_{i \in A} X_{i}
$$

Let $\left\{A_{n}, n \geqslant 1\right\}$ (we always put $A_{0}=\emptyset$ ) be a sequence of arbitrarily dependent subsets of positive integer numbers $\mathbb{N}$ that are almost surely bounded, that is, there exists a sequence of positive reals $\left\{\bar{\alpha}_{n}, n \geqslant 1\right\}$, possibly, divergent to infinity, such that

$$
\mathbf{P}\left[\sup \left\{k: k \in A_{n}\right\} \leqslant \bar{\alpha}_{n}\right]=1 .
$$

Throughout the paper, we assume that $\left\{A_{n}, n \geqslant 1\right\}$ and $\left\{X_{n}, n \geqslant 1\right\}$ are independent, that is, for every sequence $\left\{B_{n}, n \geqslant 1\right\}$ of subsets of $2^{\mathbb{N}}$ and every sequence $\left\{C_{n}, n \geqslant 1\right\}$ of measurable Borel sets on $\mathbb{R}$, for 
every $n \in \mathbb{N}$, we have

$$
\mathbf{P}\left[A_{k} \in B_{k}, X_{k} \in C_{k}, 1 \leqslant k \leqslant n\right]=\mathbf{P}\left[A_{k} \in B_{k}, 1 \leqslant k \leqslant n\right] \mathbf{P}\left[X_{k} \in C_{k}, 1 \leqslant k \leqslant n\right] .
$$

The general aim of this paper is to establish the strong law of large numbers for the sums $\left\{S\left(A_{n}\right), n \geqslant 1\right\}$. Randomly indexed sums have not been considered yet. Generally, this problem seems very difficult.

In our results, there often (although not always) arises the assumption

$$
A_{n} \subset A_{n+1} \text { a.s., } \quad n \geqslant 1 \text {. }
$$

Therefore the investigation of the behavior $\left\{S\left(A_{n}\right) / b_{n}, n \geqslant 1\right\}$ for some sequence of divergent to infinity positive reals $\left\{b_{n}, n \geqslant 1\right\}$ under (1.2) is equivalent to investigating $\left\{\sum_{i=1}^{n} S_{(i)} / b_{n}, n \geqslant 1\right\}$, where

$$
S_{(n)}=\sum_{i \in A_{n} \backslash A_{n-1}} X_{i}-\mathbf{E} \sum_{i \in A_{n} \backslash A_{n-1}} X_{i}, \quad n \geqslant 1,
$$

is the sequence of dependent random variables.

The most recent result for a sequence of dependent mixing random variables is due to Hu and Weber [4] (see also [2] and [3]). Their result, formulated in our terms, may be stated as follows.

Theorem 1. (See [4, Thm. 1.1 and Cor. 1.2].) Let $\left\{X_{n}, n \geqslant 1\right\}$ be a sequence of random variables, and let $\left\{A_{n}, n \geqslant 1\right\}$ be a sequence of random subsets of $\mathbb{N}$ such that $A_{n} \subset A_{n+1}$ a.s. $n \geqslant 1$. Let $\left\{b_{n}, n \geqslant 1\right\}$ be an increasing sequence of positive constants. Assume that there exists a constant $K$ such that, for all $n \geqslant 1$,

$$
\frac{n}{b_{n}} \leqslant K
$$

Suppose that

$$
\sum_{n=1}^{\infty} \frac{\operatorname{Var}\left(\sum_{i \in A_{n} \backslash A_{n-1}} X_{i}\right) \log ^{2} n}{b_{n}^{2}}<\infty
$$

and

$$
\sum_{k=1}^{\infty} \sup _{n \geqslant 1}\left|\operatorname{Cov}\left(\sum_{i \in A_{n+1} \backslash A_{n}} X_{i}, \sum_{i \in A_{n+k+1} \backslash A_{n+k}} X_{i}\right)\right| \frac{\log ^{2} k}{k}<\infty .
$$

Then

$$
\lim _{n \rightarrow \infty} \frac{S\left(A_{n}\right)}{b_{n}}=0 \quad \text { a.s. }
$$

The general aim of our paper is to obtain a new SLLN for $\left\{S\left(A_{n}\right) / b_{n}, n \geqslant 1\right\}$ with the following improvements:

- We assume nothing about the mixing structure type (1.5). In Example 1 and Remark 2 in Section 4, we construct the sequence $\left\{X_{n}, n \geqslant 1\right\}$ such that $\left\{S_{(n)}, n \geqslant 1\right\}$ are dependent and not satisfying (1.5) but such that for this sequence, our result holds.

- We remove assumption (1.3).

- Our results essentially weaken assumption (1.4).

- We consider both the Marcinkiewicz-Zygmund and Kolmogorov SLLNs.

- The technique of proof of our results is essentially different from that presented in papers $[3,4]$ and $[2]$. We develop the technique described in [1] and [8].

We postpone a discussion and comparison of our results with Theorem 1 to Section 4 devoted to remarks, examples, and conclusions. Section 2 contains the main results, which are proved in Section 3.

Throughout the paper, $C$ denotes the generic constants, and we always assume that $\sum_{i \in \emptyset} a_{i}=0$. 


\section{Main results}

Let $\left\{X_{n}, n \geqslant 1\right\}$ be a sequence of random variables such that $\mathbf{E}\left|X_{n}\right|<\infty, n \geqslant 1$. For an arbitrary random subset $A$ of natural numbers, we denote

$$
\begin{aligned}
V(A) & =\sum_{i \in A} X_{i}-\sum_{i \in A} \mathbf{E} X_{i}=\sum_{i \in A}\left(X_{i}-\mathbf{E} X_{i}\right), \\
Z(A) & =\sum_{i \in A} \mathbf{E} X_{i}-\mathbf{E} \sum_{i \in A} \mathbf{E} X_{i}=\sum_{i=1}^{\infty}(\mathbf{I}[i \in A]-\mathbf{P}[i \in A]) \mathbf{E} X_{i}, \\
S(A) & =\sum_{i \in A} X_{i}-\mathbf{E} \sum_{i \in A} X_{i} .
\end{aligned}
$$

Note that if $A$ is independent of $\left\{X_{n}, n \geqslant 1\right\}$, then

$$
S(A)=V(A)+Z(A) .
$$

Theorem 2. Let $\left\{X_{n}, n \geqslant 1\right\}$ be a sequence of independent random variables such that $\mathbf{E}\left|X_{n}\right|<\infty, n \geqslant 1$, and let $\left\{A_{n}, n \geqslant 0\right\}$ be a sequence of random subsets of $\mathbb{N}\left(A_{o}=\emptyset\right)$ independent of $\left\{X_{n}, n \geqslant 1\right\}$ and satisfying (1.1). Let $\left\{b_{n}, n \geqslant 1\right\}$ be a nondecreasing unbounded sequence of positive reals. Introduce the following conditions:

(a) for $q>1$,

$$
\sum_{n=1}^{\infty} \mathbf{E} \sum_{j \in A_{n} \backslash A_{n-1}} \mathbf{E}\left|X_{j}-\mathbf{E} X_{j}\right|^{2 q} \sum_{k=n+1}^{\infty} \frac{\left|A_{k}\right|^{q-1}-\left|A_{k-1}\right|^{q-1}}{b_{k}^{2 q}}<\infty
$$

(b) for $q \geqslant 1$,

$$
\sum_{n=1}^{\infty} \mathbf{E} \sum_{j \in A_{n} \backslash A_{n-1}} \mathbf{E}\left|X_{j}-\mathbf{E} X_{j}\right|^{2 q} \frac{\left|A_{n}\right|^{q-1}}{b_{n}^{2 q}}<\infty
$$

(c)

$$
A_{n} \subset A_{n+1} \text { a.s., } \quad n \geqslant 1 ;
$$

(d) for every $\epsilon>0$,

$$
\begin{gathered}
\sum_{n=1}^{\infty}\left(\mathrm{e}^{-b_{n} \epsilon+\mathbf{E} \sum_{i \in A_{n}} X_{i}} \mathbf{E} \prod_{i \in A_{n}} \mathrm{e}^{-\mathbf{E} X_{i}}\right)<\infty, \\
\sum_{n=1}^{\infty}\left(\mathrm{e}^{-b_{n} \epsilon-\mathbf{E} \sum_{i \in A_{n}} X_{i}} \mathbf{E} \prod_{i \in A_{n}} \mathrm{e}^{\mathbf{E} X_{i}}\right)<\infty .
\end{gathered}
$$

If for some $q \geqslant 1, \mathbf{E}\left|X_{n}\right|^{2 q}<\infty, n \geqslant 1$, and (a)-(c) are satisfied, then

$$
\lim _{n \rightarrow \infty} \frac{V\left(A_{n}\right)}{b_{n}}=0 \quad \text { a.s. }
$$

If (d) is satisfied, then

$$
\lim _{n \rightarrow \infty} \frac{Z\left(A_{n}\right)}{b_{n}}=0 \quad \text { a.s. }
$$


If for some $q \geqslant 1, \mathbf{E}\left|X_{n}\right|^{2 q}<\infty, n \geqslant 1$, and (a)-(d) are satisfied, then

$$
\lim _{n \rightarrow \infty} \frac{S\left(A_{n}\right)}{b_{n}}=0 \quad \text { a.s. }
$$

Corollary 1. Let $\left\{X, X_{n}, n \geqslant 1\right\}$ be a sequence of independent identically distributed random variables such that $\mathbf{E}|X|<\infty$, and let $\left\{A_{n}, n \geqslant 0\right\}$ be a sequence of random subsets of $\mathbb{N}\left(A_{o}=\emptyset\right)$ independent of $\left\{X_{n}, n \geqslant 1\right\}$ and satisfying (1.1). Let $\left\{b_{n}, n \geqslant 1\right\}$ be a nondecreasing unbounded sequence of positive reals. Introduce the following conditions:

(i)

$$
A_{n} \subset A_{n+1} \text { a.s., } \quad n \geqslant 1
$$

(ii) for $q>1$,

$$
\sum_{n=1}^{\infty} \mathbf{E}\left(\left(\left|A_{n}\right|-\left|A_{n-1}\right|\right) \sum_{k=n+1}^{\infty} \frac{\left|A_{k}\right|^{q-1}-\left|A_{k-1}\right|^{q-1}}{b_{k}^{2 q}}\right)<\infty
$$

(iii) for $q \geqslant 1$,

$$
\sum_{n=1}^{\infty} \mathbf{E} \frac{\left(\left|A_{n}\right|-\left|A_{n-1}\right|\right)\left|A_{n}\right|^{q-1}}{b_{n}^{2 q}}<\infty
$$

(iv) for every $\epsilon>0$,

$$
\sum_{n=1}^{\infty} \mathrm{e}^{-b_{n} \epsilon} \mathbf{E}\left(\mathrm{e}^{|| A_{n}|-E| A_{n} \|}\right)^{\mathbf{E} X}<\infty .
$$

If $\mathbf{E}|X|^{2 q}<\infty, q \geqslant 1$, and (i)-(iii) are satisfied, then

$$
\lim _{n \rightarrow \infty} \frac{V\left(A_{n}\right)}{b_{n}}=0 \quad \text { a.s. }
$$

If (iv) is satisfied, then

$$
\lim _{n \rightarrow \infty} \frac{Z\left(A_{n}\right)}{b_{n}}=0 \quad \text { a.s. }
$$

If $\mathbf{E}|X|^{2 q}<\infty, q \geqslant 1$, and (i)-(iv) are satisfied, then

$$
\lim _{n \rightarrow \infty} \frac{S\left(A_{n}\right)}{b_{n}}=0 \quad \text { a.s. }
$$

It is worth noting that condition (iv) of the corollary is satisfied when

$$
\lim _{n \rightarrow \infty} \frac{\log \mathbf{E}\left(\mathrm{e}^{|| A_{n}|-\mathbf{E}| A_{n} \|}\right)^{\mathbf{E} X}}{b_{n}}=0
$$

and, for every $\epsilon>0$,

$$
\sum_{n=1}^{\infty} \mathrm{e}^{-\epsilon b_{n}}<\infty
$$

Let us now consider the case where $\left\{X_{n}, n \geqslant 1\right\}$ are arbitrary dependent but identically distributed. 
Theorem 3. Let $\left\{X, X_{n}, n \geqslant 1\right\}$ be a sequence of arbitrary dependent identically distributed random variables such that $\mathbf{E}|X|<\infty$, and let $\left\{A_{n}, n \geqslant 0\right\}$ be a sequence of a random subsets of $\mathbb{N}\left(A_{o}=\emptyset\right)$ independent of $\left\{X, X_{n}, n \geqslant 1\right\}$ such that

$$
A_{n-1} \subset A_{n} \text { a.s., } \quad n \geqslant 1 .
$$

Suppose that $b_{0}=0$ and $\left\{b_{n}, n \geqslant 1\right\}$ is a nondecreasing divergent to infinity sequence of positive constants and

$$
\begin{gathered}
\sum_{k=1}^{\infty} b_{k} P\left[b_{k-1}<|X-\mathbf{E} X| \leqslant b_{k}\right] \sum_{n=1}^{\infty} \frac{\mathbf{E}\left|\left(A_{n} \backslash A_{n-1}\right) \cap[k, \infty)\right|}{b_{n}}<\infty, \\
\liminf _{k \rightarrow \infty} \frac{b_{k}}{k} \sum_{n=1}^{\infty} \frac{\mathbf{E}\left|\left(A_{n} \backslash A_{n-1}\right) \cap[k, \infty)\right|}{b_{n}}>0 .
\end{gathered}
$$

Assume additionally that, for every $\epsilon>0$, we have

$$
\sum_{n=1}^{\infty} \mathrm{e}^{-b_{n} \epsilon}\left(\mathrm{e}^{\mathbf{E} X \mathbf{E}\left|A_{n}\right|} \mathbf{E}\left(\mathrm{e}^{-\mathbf{E} X}\right)^{\left|A_{n}\right|}+\mathrm{e}^{-\mathbf{E} X \mathbf{E}\left|A_{n}\right|} \mathbf{E}\left(\mathrm{e}^{\mathbf{E} X}\right)^{\left|A_{n}\right|}\right)<\infty .
$$

Then

$$
\lim _{n \rightarrow \infty} \frac{S\left(A_{n}\right)}{b_{n}}=0 \quad \text { a.s. }
$$

It is easy to check that in the case of $A_{n}=\{1,2, \ldots, n\}, n \geqslant 1$, from Theorem 3 we obtain Theorem 2.1 of [8].

\section{Proofs}

In this section, for an arbitrary set $A \subset \mathbb{N}$ and for an arbitrary sequence of random variables $\left\{X_{n}, n \geqslant 1\right\}$, we will use notation (2.1). Because $V\left(A_{n}\right)$ may be written as the sum of random variables, the classical HájekRényi inequality holds.

Lemma 1. (See Hájek-Rényi-type maximal inequality, [1, Thm. 1.1].) Let $\left\{A_{n}, n \geqslant 0\right\}, A_{0}=\emptyset$, be an a.s. increasing sequence of random subsets of $\mathbb{N}$ satisfying (1.1) and independent of the sequence of random variables $\left\{X_{n}, n \geqslant 1\right\}$. Let $\beta_{1}, \beta_{2}, \ldots$ be a nondecreasing sequence of positive numbers. Let $\alpha_{1}, \alpha_{2}, \ldots$ be nonnegative numbers. Let $r$ be a fixed positive number. Assume that for each $m$ with $1 \leqslant m \leqslant n$,

$$
\mathbf{E}\left[\max _{1 \leqslant l \leqslant m}\left|V\left(A_{l}\right)\right|\right]^{r} \leqslant \sum_{l=1}^{m} \alpha_{l} .
$$

Then

$$
\mathbf{E}\left[\max _{1 \leqslant l \leqslant n}\left|\frac{V\left(A_{l}\right)}{\beta_{l}}\right|\right]^{r}<4 \sum_{l=1}^{n} \frac{\alpha_{l}}{\beta_{l}^{r}} .
$$

Lemma 2. (See [1, Thm. 2.1].) Let $\left\{A_{n}, n \geqslant 0\right\}$ be a sequence of a.s. increasing random subsets of $\mathbb{N}$ satisfying (1.1):

$$
A_{n}: \Omega \rightarrow 2^{\mathbb{N}}, \quad A_{n} \subset A_{n+1} \text { a.s., } \quad n \geqslant 1 .
$$


Let $\left\{b_{n}, n \geqslant 1\right\}$ be a nondecreasing unbounded sequence of positive numbers, and let $\left\{\alpha_{n}, n \geqslant 1\right\}$ be a sequence of nonnegative numbers. Let $r$ be a fixed positive number. Assume that for each $n \geqslant 1$,

$$
\mathbf{E}\left[\max _{1 \leqslant l \leqslant n}\left|V\left(A_{l}\right)\right|\right]^{r} \leqslant \sum_{l=1}^{n} \alpha_{l} .
$$

If $\sum_{l=1}^{\infty} \alpha_{l} / b_{l}^{r}<\infty$, then

$$
\lim _{n \rightarrow \infty} \frac{V\left(A_{n}\right)}{b_{n}}=0 \quad \text { a.s. }
$$

Proof of Theorem 2. We first consider convergence (2.7). The proof essentially runs similarly as that of Corollary 3.1 in [1]. We only remark that in our case the Doob inequality is

$$
\begin{aligned}
\mathbf{E}\left[\max _{1 \leqslant k \leqslant n}\left|V\left(A_{k}\right)\right|\right]^{2 q} & =\sum^{\prime} \mathbf{E}\left[\max _{1 \leqslant k \leqslant n}\left|V\left(B_{k}\right)\right|\right]^{2 q} \mathbf{P}\left[A_{k}=B_{k}, 1 \leqslant k \leqslant n\right] \\
& \leqslant\left(\frac{2 q}{2 q-1}\right)^{2 q} \sum^{\prime} \mathbf{E}\left[\left|V\left(B_{n}\right)\right|\right]^{2 q} \mathbf{P}\left[A_{k}=B_{k}, 1 \leqslant k \leqslant n\right] \\
& \leqslant\left(\frac{2 q}{2 q-1}\right)^{2 q} \mathbf{E}\left|V\left(A_{n}\right)\right|^{2 q},
\end{aligned}
$$

where $\sum^{\prime}$ is the sum taken over all possible sets $\left\{B_{k}, 1 \leqslant k \leqslant n\right\}$ such that $B_{i} \subset B_{i+1}, 1 \leqslant i \leqslant n-1$, and $B_{n} \subset\left\{1,2, \ldots, \bar{\alpha}_{n}\right\}$ (cf. (1.1)), and the Burkholder inequality is

$$
\mathbf{E}\left[\left|V\left(A_{n}\right)\right|\right]^{2 q} \leqslant c_{q} \mathbf{E}\left(\sum_{j \in A_{n}}\left(X_{j}-\mathbf{E} X_{j}\right)^{2}\right)^{q} .
$$

Applying the Hölder inequality for $q>1$, we get

$$
\mathbf{E}\left[\left|V\left(A_{n}\right)\right|\right]^{2 q} \leqslant c_{q} \mathbf{E}\left(\sum_{j \in A_{n}}\left|X_{j}-\mathbf{E} X_{j}\right|^{2 q}\left|A_{n}\right|^{q-1}\right) .
$$

Now putting, for $n \geqslant 1$,

$$
\alpha_{n}= \begin{cases}c(q)\left(\mathbf{E} \sum_{j \in A_{n} \backslash A_{n-1}} \mathbf{E}\left|X_{j}-\mathbf{E} X_{j}\right|^{2 q}\left|A_{n}\right|^{q-1}\right. & \\ \left.+\mathbf{E} \sum_{j \in A_{n-1}} \mathbf{E}\left|X_{j}-\mathbf{E} X_{j}\right|^{2 q}\left(\left|A_{n}\right|^{q-1}-\left|A_{n-1}\right|^{q-1}\right)\right) & \text { if } q>1, \\ c(q) \mathbf{E} \sum_{j \in A_{n} \backslash A_{n-1}} \operatorname{Var}\left(X_{j}\right) & \text { if } q=1,\end{cases}
$$

where $c(q)=c_{q}(2 q /(2 q-1))^{2 q}$, we get

$$
\mathbf{E}\left[\max _{1 \leqslant k \leqslant n}\left|V\left(A_{k}\right)\right|\right]^{2 q} \leqslant \sum_{j=1}^{n} \alpha_{j}, \quad n \geqslant 1 .
$$

Thus (a), (b), and Lemma 2 end the proof of (2.7).

To prove (2.8), it suffices to show that for all $\varepsilon>0$,

$$
\sum_{n=1}^{\infty} \mathbf{P}\left[\left|\frac{Z\left(A_{n}\right)}{b_{n}}\right|>\varepsilon\right]<\infty
$$


Notice that by the Chebyshev exponential inequality we have

$$
\sum_{n=1}^{\infty} \mathbf{P}\left[Z\left(A_{n}\right)>\varepsilon b_{n}\right] \leqslant \sum_{n=1}^{\infty} \frac{\mathbf{E} \mathrm{e}^{Z\left(A_{n}\right)}}{\mathrm{e}^{\varepsilon b_{n}}}=\sum_{n=1}^{\infty} \mathrm{e}^{-\varepsilon b_{n}} \mathbf{E} \mathrm{e}^{\sum_{i=1}^{\infty}\left(\mathbf{I}\left[i \in A_{n}\right]-p_{n, i}\right) \mathbf{E} X_{i}},
$$

where $p_{n, i}=\mathbf{P}\left[i \in A_{n}\right], i, n \geqslant 1$. Next, taking into account that

$$
\sum_{i=1}^{\infty} p_{n, i} \mathbf{E} X_{i}=\mathbf{E} \sum_{i \in A_{n}} X_{i}, \quad n \geqslant 1,
$$

and by the property of the indicator function we have

$$
\begin{aligned}
\mathbf{E} \mathrm{e}^{\sum_{i=1}^{\infty}\left(\mathbf{I}\left[i \in A_{n}\right]-p_{n, i}\right) \mathbf{E} X_{i}} & =\mathrm{e}^{-\sum_{i=1}^{\infty} p_{n, i} \mathbf{E} X_{i}} \mathbf{E} \mathrm{e}^{\sum_{i=1}^{\infty} \mathbf{I}\left[i \in A_{n}\right] X_{i}} \\
& =\mathrm{e}^{-\mathbf{E} \sum_{i \in A_{n}} X_{i}} \mathbf{E} \prod_{i \in A_{n}} \mathrm{e}^{\mathbf{E} X_{i}} .
\end{aligned}
$$

Thus from (2.6) we obtain

$$
\sum_{n=1}^{\infty} \mathbf{P}\left[Z\left(A_{n}\right)>\varepsilon b_{n}\right]<\infty
$$

Similarly, we have

$$
\mathbf{E} \mathrm{e}^{-\sum_{i=1}^{\infty}\left(\mathbf{I}\left[i \in A_{n}\right]-p_{n, i}\right) \mathbf{E} X_{i}}=\mathrm{e}^{\mathbf{E} \sum_{i \in A_{n}} X_{i}} \mathbf{E} \prod_{i \in A_{n}} \mathrm{e}^{-\mathbf{E} X_{i}}
$$

and from (2.5) we have

$$
\sum_{n=1}^{\infty} \mathbf{P}\left[Z\left(A_{n}\right)<-\varepsilon b_{n}\right]<\infty
$$

Proof of Theorem 3. Let us first prove that

$$
\lim _{n \rightarrow \infty} \frac{V\left(A_{n}\right)}{b_{n}}=0 \quad \text { a.s. }
$$

Because

$$
\begin{aligned}
V\left(A_{n}\right)= & \sum_{i \in A_{n}}\left(X_{i}-\mathbf{E} X_{i}\right) \mathbf{I}\left[\left|X_{i}-\mathbf{E} X_{i}\right|>b_{i}\right] \\
& +\sum_{k=1}^{n} \sum_{i \in A_{k} \backslash A_{k-1}}\left(X_{i}-\mathbf{E} X_{i}\right) \mathbf{I}\left[\left|X_{i}-\mathbf{E} X_{i}\right| \leqslant b_{i}\right],
\end{aligned}
$$

to obtain (3.1), it suffices to prove that

$$
\sum_{i=1}^{\infty}\left|X_{i}-\mathbf{E} X_{i}\right| \mathbf{I}\left[\left|X_{i}-\mathbf{E} X_{i}\right|>b_{i}\right]<\infty \quad \text { a.s. }
$$

and (by the Kronecker lemma)

$$
\sum_{n=1}^{\infty} \frac{1}{b_{n}} \sum_{i \in A_{n} \backslash A_{n-1}}\left|X_{i}-\mathbf{E} X_{i}\right| \mathbf{I}\left[\left|X_{i}-\mathbf{E} X_{i}\right| \leqslant b_{i}\right]<\infty \quad \text { a.s. }
$$


Proof of (3.2). From (2.10) and (2.11) we may conclude that

$$
\sum_{k=1}^{\infty} \mathbf{P}\left[|X-\mathbf{E} X|>b_{k}\right]=\sum_{k=1}^{\infty} k \mathbf{P}\left[b_{k-1}<|X-\mathbf{E} X| \leqslant b_{k}\right]-\mathbf{P}[|X-\mathbf{E} X| \neq 0]<\infty .
$$

Thus by the Borel-Cantelli lemma there exists the positive integer-valued random variable $Y$ such that

and therefore

$$
\mathbf{P}\left[\bigcup_{n \geqslant Y}\left[\left|X_{n}-\mathbf{E} X_{n}\right|>b_{n}\right]\right]=0
$$

$$
\sum_{i=1}^{\infty}\left|X_{i}-\mathbf{E} X_{i}\right| \mathbf{I}\left[\left|X_{i}-\mathbf{E} X_{i}\right|>b_{i}\right]=\sum_{i \leqslant Y}\left|X_{i}-\mathbf{E} X_{i}\right| \mathbf{I}\left[\left|X_{i}-\mathbf{E} X_{i}\right|>b_{i}\right]<\infty \quad \text { a.s. }
$$

which ends the proof of (3.2).

Proof of (3.3). From (2.10) we have

$$
\begin{aligned}
\sum_{n=1}^{\infty} & \frac{1}{b_{n}} \mathbf{E} \sum_{i \in A_{n} \backslash A_{n-1}}\left|X_{i}-\mathbf{E} X_{i}\right| \mathbf{I}\left[\left|X_{i}-\mathbf{E} X_{i}\right| \leqslant b_{i}\right] \\
& =\sum_{n=1}^{\infty} \frac{1}{b_{n}} \mathbf{E} \sum_{i \in A_{n} \backslash A_{n-1}} \sum_{k=1}^{i}\left|X_{i}-\mathbf{E} X_{i}\right| \mathbf{I}\left[b_{k-1}<\left|X_{i}-\mathbf{E} X_{i}\right| \leqslant b_{k}\right] \\
& \leqslant \sum_{n=1}^{\infty} \frac{1}{b_{n}} \mathbf{E} \sum_{i \in A_{n} \backslash A_{n-1}} \sum_{k=1}^{i} b_{k} \mathbf{P}\left[b_{k-1}<|X-\mathbf{E} X| \leqslant b_{k}\right] \\
& =\sum_{n=1}^{\infty} \frac{1}{b_{n}} \sum_{k=1}^{\infty} b_{k} \mathbf{P}\left[b_{k-1}<|X-\mathbf{E} X| \leqslant b_{k}\right] \mathbf{E} \sum_{i \in A_{n} \backslash A_{n-1}, i \geqslant k} 1 \\
& =\sum_{n=1}^{\infty} \frac{1}{b_{n}} \sum_{k=1}^{\infty} b_{k} \mathbf{P}\left[b_{k-1}<|X-\mathbf{E} X| \leqslant b_{k}\right] \mathbf{E}\left|\left(A_{n} \backslash A_{n-1}\right) \cap[k, \infty)\right| \\
& =\sum_{k=1}^{\infty} b_{k} \mathbf{P}\left[b_{k-1}<|X-\mathbf{E} X| \leqslant b_{k}\right] \sum_{n=1}^{\infty} \frac{\mathbf{E}\left|\left(A_{n} \backslash A_{n-1}\right) \cap[k, \infty)\right|}{b_{n}} \\
& <\infty,
\end{aligned}
$$

and since $L^{1}$ bounded series are almost surely bounded, we also get (3.3).

To prove Theorem 3, we need to show that

$$
\lim _{n \rightarrow \infty} \frac{Z\left(A_{n}\right)}{b_{n}}=0 \quad \text { a.s., }
$$

but because complete convergence implies almost sure convergence, it suffices to prove that for every $\epsilon>0$,

$$
\sum_{n=1}^{\infty} \mathbf{P}\left[\left|Z\left(A_{n}\right)\right|>\epsilon b_{n}\right]<\infty
$$


By the Chebyshev exponential inequality we have

$$
\begin{aligned}
\sum_{n=1}^{\infty} \mathbf{P}\left[Z\left(A_{n}\right)>\varepsilon b_{n}\right] & \leqslant \sum_{n=1}^{\infty} \mathrm{e}^{-\varepsilon b_{n}} \mathbf{E} \mathrm{e}^{\mathbf{E} X\left[\sum_{i=1}^{\infty}\left(\mathbf{I}\left[i \in A_{n}\right]-p_{n, i}\right)\right]}, \\
\sum_{n=1}^{\infty} \mathbf{P}\left[Z\left(A_{n}\right)<-\varepsilon b_{n}\right] & \leqslant \sum_{n=1}^{\infty} \mathrm{e}^{-\varepsilon b_{n}} \mathbf{E} \mathrm{e}^{\mathbf{E} X\left[\sum_{i=1}^{\infty}\left(p_{n, i}-\mathbf{I}\left[i \in A_{n}\right]\right)\right]},
\end{aligned}
$$

where $p_{n, i}=P\left[i \in A_{n}\right], n \geqslant 1,1 \leqslant i \leqslant \bar{\alpha}_{n}$. Furthermore, because $\left|A_{n}\right|=\sum_{i=1}^{\infty} \mathbf{I}\left[i \in A_{n}\right], \mathbf{E}\left|A_{n}\right|=$ $\sum_{i=1}^{\infty} p_{n, i}$, and (3.4) follows from (2.12).

\section{Remarks and examples}

Remark 1. Let us remark that the assumption $\mathbf{E}|X|<\infty$ in Theorem 3 is superfluous. Replacing $X-\mathbf{E} X$ by $X$ in the proof, we obtain the SLLN of the following form:

$$
\lim _{n \rightarrow \infty} \frac{\sum_{i \in A_{n}} X_{i}}{b_{n}}=0
$$

However, in this paper, we emphasize the influence of decomposition (2.2) on SLLN, and therefore we assume the existence of the first moment of $X$.

Let us consider the increments of terms $V\left(A_{n}\right), Z\left(A_{n}\right), S\left(A_{n}\right)$, that is, the terms $V_{(n)}=V\left(A_{n+1} \backslash A_{n}\right)$, $Z_{(n)}=Z\left(A_{n+1} \backslash A_{n}\right), S_{(n)}=S\left(A_{n+1} \backslash A_{n}\right), n \geqslant 1$, respectively.

Remark 2. Let $\left\{X_{n}, n \geqslant 1\right\}$ be a sequence of independent random variables, and let $\left\{A_{n}, n \geqslant 1\right\}$ be a sequence of almost surely increasing $2^{\mathbb{N}}$-valued random sets independent of $\left\{X_{n}, n \geqslant 1\right\}$. We have:

(a) The increments of $V\left(A_{n}\right)$ are uncorrelated.

(b) The increments of $Z\left(A_{n}\right)$ and $S\left(A_{n}\right)$ may be correlated.

(c) The increments of $V\left(A_{n}\right), Z\left(A_{n}\right)$, and $S\left(A_{n}\right)$ may be dependent.

Proof of Remark 2(a). Because $\left\{A_{n}, n \geqslant 1\right\}$ and $\left\{X_{n}, n \geqslant 1\right\}$ are independent, and $\left\{X_{n}, n \geqslant 1\right\}$ are independent random variables, we have

$$
\begin{aligned}
\operatorname{Cov}\left(V_{(n)}, V_{(n+k)}\right)= & \operatorname{Cov}\left(\sum_{j \in A_{n+1} \backslash A_{n}}\left(X_{j}-\mathbf{E} X_{j}\right), \sum_{j \in A_{n+k+1} \backslash A_{n+k}}\left(X_{j}-\mathbf{E} X_{j}\right)\right) \\
= & \sum^{\prime} \sum_{j \in B \backslash A} \sum_{k \in D \backslash C} \mathbf{E}\left(X_{j}-\mathbf{E} X_{j}\right)\left(X_{k}-\mathbf{E} X_{k}\right) \\
& \times \mathbf{P}\left[A_{n}=B_{1}, A_{n+1}=B_{2}, A_{n+k}=B_{3}, A_{n+k+1}=B_{4}\right]=0,
\end{aligned}
$$

where the summation in $\sum^{\prime}$ is taken over all possible sets $\left\{B_{k}, 1 \leqslant k \leqslant 4\right\}$ such that $B_{i} \subset B_{i+1}, i=1,2,3$, and $B_{4} \subset\left\{1,2, \ldots, \bar{\alpha}_{n+k+1}\right\}$ (cf. (1.1)).

For points (b) and (c) of Remark 2, we construct the following example.

Example 1. Let $\left\{X_{n}, n \geqslant 1\right\}$ be a sequence of independent Gaussian $N\left(\mu_{n}, \sigma_{n}\right)$ random variables defined on the probability space $([0,1], \mathcal{B}, \lambda)(\mathcal{B}$ denotes the family of Borel subsets of $[0,1]$, and $\lambda$ is the Lebesgue measure on $[0,1])$. On the same probability space $([0,1], \mathcal{B}, \lambda)$, we will define the sequence of random sets 
$\left\{A_{n}, n \geqslant 1\right\}$ and expand the definitions of $\left\{X_{n}, A_{n}, n \geqslant 1\right\}$ on the product space $\left([0,1]^{2}, \mathcal{B} \times \mathcal{B}, \lambda \times \lambda\right)$ such that $\left\{X_{n}, n \geqslant 1\right\}$ and $\left\{A_{n}, n \geqslant 1\right\}$ will be independent $\left(X_{n}\left(\omega_{1}, \omega_{2}\right)=X_{n}\left(\omega_{1}\right), A_{n}\left(\omega_{1}, \omega_{2}\right)=A_{n}\left(\omega_{2}\right)\right)$. For some $0<p<1$, we define

$$
I_{n}= \begin{cases}{[0, p)} & \text { if } n \text { is even } \\ {[p, 1]} & \text { if } n \text { is odd }\end{cases}
$$

and let

$$
A_{n}= \begin{cases}\{1,2, \ldots, n, n+1\}, & \omega \in[0,1] \times I_{n}, \\ \{1,2, \ldots, n, n+2\}, & \omega \in[0,1] \times\left([0,1] \backslash I_{n}\right) .\end{cases}
$$

Obviously $A_{n} \subset A_{n+1}$ a.s. $n \geqslant 1$. In the case where $n$ is even, $n+1$ is odd, and $I_{n}=[0, p), I_{n+1}=[p, 1]$. Therefore for $\omega \in[0,1] \times[0, p), A_{n}(\omega)$ and $A_{n+1}(\omega)$ are defined by the first and second formulas in (4.1), respectively. Thus

$$
\begin{aligned}
A_{n+1}(\omega) \backslash A_{n}(\omega) & =\{1,2, \ldots, n, n+1, n+3\} \backslash\{1,2, \ldots, n, n+1\} \\
& =\{n+3\},
\end{aligned}
$$

whereas for $\omega \in[0,1] \times[p, 1]$ we take $A_{n+1}(\omega)$ defined by first formula and $A_{n}(\omega)$ by the second formula in (4.1). Thus

$$
\begin{aligned}
A_{n+1}(\omega) \backslash A_{n}(\omega) & =\{1,2, \ldots, n, n+1, n+2\} \backslash\{1,2, \ldots, n, n+2\} \\
& =\{n+1\} .
\end{aligned}
$$

Proceeding similarly for the case of odd $n$, we establish

$$
A_{n+1}(\omega) \backslash A_{n}(\omega)= \begin{cases}\{n+1\}, & \omega \in[0,1] \times\left([0,1] \backslash I_{n}\right), \\ \{n+3\}, & \omega \in[0,1] \times I_{n} .\end{cases}
$$

Proof of items (b) and (c) of Remark 2. In Example 1, from the independency of $\left\{X_{n}, n \geqslant 1\right\}$ and $\left\{A_{n}, n \geqslant 1\right\}$ we have

$$
\begin{aligned}
\operatorname{Cov}\left(S_{(n)}, S_{(n+k)}\right) & =\operatorname{Cov}\left(\sum_{j \in A_{n+1} \backslash A_{n}} X_{j}, \sum_{j \in A_{n+k+1} \backslash A_{n+k}} X_{j}\right) \\
& =(-1)^{I[k \text { is odd }]} p(1-p)\left(\mu_{n+3}-\mu_{n+1}\right)\left(\mu_{n+k+3}-\mu_{n+k+1}\right),
\end{aligned}
$$

where $I[B]$ is the indicator (or characteristic function) of event $B$. Now if $\mu_{n}=n \delta$ for some $\delta>0$, then we have

$$
\operatorname{Cov}\left(S_{(n)}, S_{(n+k)}\right)=(-1)^{I[k \text { is odd }]} 4 p(1-p) \delta^{2} \neq 0 .
$$

By similar computations we get

$$
\operatorname{Cov}\left(Z_{(n)}, Z_{(n+k)}\right)=(-1)^{I[k \text { is odd }]} 4 p(1-p) \delta^{2} \neq 0,
$$

which ends the proof of (b).

For (c), let us assume that $k$ is even and $\mu_{n}=0$. Then

$$
\begin{aligned}
\phi_{V_{(n)}, V_{(n+k)}}(t, s) & =\mathbf{P}\left[I_{n}\right] \mathrm{e}^{-t^{2} \sigma_{n+3}^{2} / 2-s^{2} \sigma_{n+k+3}^{2} / 2}+\left(1-\mathbf{P}\left[I_{n}\right]\right) \mathrm{e}^{-t^{2} \sigma_{n+1}^{2} / 2-s^{2} \sigma_{n+k+1}^{2} / 2}, \\
\phi_{V_{(n)}}(t) & =\mathbf{P}\left[I_{n}\right] \mathrm{e}^{-t^{2} \sigma_{n+3}^{2} / 2}+\left(1-\mathbf{P}\left[I_{n}\right]\right) \mathrm{e}^{-t^{2} \sigma_{n+1}^{2} / 2}, \\
\phi_{V_{(n+k)}}(s) & =\mathbf{P}\left[I_{n}\right] \mathrm{e}^{-s^{2} \sigma_{n+k+3}^{2} / 2}+\left(1-\mathbf{P}\left[I_{n}\right]\right) \mathrm{e}^{-s^{2} \sigma_{n+k+1}^{2} / 2},
\end{aligned}
$$


where $\phi_{X}(t)$ denotes the characteristic function of $X$. Now we put $\sigma_{n}^{2}=\log (\mathrm{e} n /(n+1)), t=s=\sqrt{2}$, $p=0.5$ (note that $\sigma_{n} \rightarrow 1$, as $n \rightarrow \infty$ ). Then we have

$$
\begin{aligned}
\phi_{V_{(n)}, V_{(n+k)}}(\sqrt{2}, \sqrt{2})-\phi_{V_{(n)}}(\sqrt{2}) \phi_{V_{(n+k)}}(\sqrt{2}) \\
=\frac{1}{2 \mathrm{e}^{2}}\left(\frac{(n+4)(n+k+4)}{(n+3)(n+k+3)}+\frac{(n+2)(n+k+2)}{(n+1)(n+k+1)}\right) \\
\quad-\frac{1}{4 \mathrm{e}^{2}}\left(\frac{n+4}{n+3}+\frac{n+2}{n+1}\right)\left(\frac{n+k+4}{n+k+3}+\frac{n+k+2}{n+k+1}\right) \\
=\frac{1}{4 \mathrm{e}^{2}}\left(\frac{1}{n+k+1}-\frac{1}{n+k+3}\right)\left(\frac{1}{n+1}-\frac{1}{n+3}\right) \\
=\frac{1}{\mathrm{e}^{2}(n+1)(n+3)(n+k+1)(n+k+3)} \neq 0 .
\end{aligned}
$$

Obviously, correlated random variables are dependent, and thus statement (c) follows from (b).

Remark 3. Defining $\left\{X_{n}, A_{n}, n \geqslant 1\right\}$ as in Example 1 with $\mu_{n}=n \delta, \sigma_{n}=1, n \geqslant 1, \delta>0$, Theorem 1 fails for every choice of the sequence $\left\{b_{n}, n \geqslant 1\right\}$, whereas if for some $q \geqslant 1$,

$$
\begin{gathered}
\sum_{n=1}^{\infty} \sum_{k=n}^{\infty} \frac{(k+1)^{q-1}-k^{q-1}}{b_{k}^{2 q}}<\infty \\
\sum_{n=1}^{\infty} \frac{n^{q-1}}{b_{n}^{2 q}}<\infty \\
\sum_{n=1}^{\infty} \mathrm{e}^{-\epsilon b_{n}}<\infty
\end{gathered}
$$

then (2.7)-(2.9) hold.

Proof. We note that when $X_{j} \sim N(j \delta, 1), X_{j}-\mathbf{E} X_{j} \sim N(0,1)$ and $\mathbf{E}\left|X_{j}-\mathbf{E} X_{j}\right|^{2 q}=\kappa_{q}$, say. Then we have

$$
\sum_{k=1}^{\infty} \sup _{n \geqslant 1}\left|\operatorname{Cov}\left(\sum_{i \in A_{n+1} \backslash A_{n}} X_{i}, \sum_{i \in A_{n+k+1} \backslash A_{n+k}} X_{i}\right)\right| \frac{\log ^{2} k}{k}=4 p(1-p) \delta^{2} \sum_{n=1}^{\infty} \frac{\log ^{2} n}{n}=\infty
$$

so that (1.5) fails. Now we show that there exists a sequence $\left\{b_{n}, n \geqslant 1\right\}$ such that the assumptions of Theorem 2 hold. Because

$$
\begin{gathered}
\mathbf{E} \sum_{i \in A_{n}} X_{i}=\frac{1}{2}((n+1)(n+2)+2) \delta-\mathbf{P}\left[I_{n}\right] \delta, \\
\mathbf{E} \prod_{i \in A_{n}} \mathrm{e}^{ \pm \mathbf{E} X_{i}}=\mathrm{e}^{ \pm(n+1)(n+2) \delta / 2}\left(\mathbf{P}\left[I_{n}\right]+\left(1-\mathbf{P}\left[I_{n}\right]\right) \mathrm{e}^{ \pm \delta}\right),
\end{gathered}
$$

(2.5) and (2.6) hold if and only if $\sum_{n=1}^{\infty} \mathrm{e}^{-\epsilon b_{n}}<\infty$. Conditions (2.3) and (2.4) are reduced to those (4.2) and (4.3), respectively. For example, we may put $b_{n}=n, q=2$, and then (4.2)-(4.4) are fulfilled. 
Open Access. This article is licensed under a Creative Commons Attribution 4.0 International License, which permits use, sharing, adaptation, distribution and reproduction in any medium or format, as long as you give appropriate credit to the original author(s) and the source, provide a link to the Creative Commons licence, and indicate if changes were made. The images or other third party material in this article are included in the article's Creative Commons licence, unless indicated otherwise in a credit line to the material. If material is not included in the article's Creative Commons licence and your intended use is not permitted by statutory regulation or exceeds the permitted use, you will need to obtain permission directly from the copyright holder. To view a copy of this licence, visit http://creativecommons.org/licenses/by/4.0/

\section{References}

1. I. Fazekas and O. Klesov, A general approach to the strong law of large numbers, Theory Probab. Appl., 45(3):436449, 2001.

2. T.-C. Hu, A. Rosalsky, and A. Volodin, On convergence properties of sums of dependent random variables under second moment and covariance restrictions, Stat. Probab. Lett., 78(14):1999-2005, 2008.

3. T.-C. Hu and R.L. Taylor, On the strong law for arrays and for the bootstrap mean and variance, Int. J. Math. Math. Sci., 20(2):375-382, 1997.

4. T.-C. Hu and N.C. Weber, A note on strong convergence of sums of dependent random variables, J. Probab. Stat., 2009:873274, 2009.

5. G. Matheron, Random Sets and Integral Geometry, Wiley Ser. Probab. Math. Stat., Wiley, New York, 1975.

6. I. Molchanov, Theory of Random Sets, Probab. Appl., Springer, London, 2005.

7. H.T. Nguyen, An Introduction to Random Sets, Chapman \& Hall/CRC, Boca Raton, FL, 2006.

8. A. Rosalsky and G. Stoica, On the strong law of large numbers for identically distributed random variables irrespective of their joint distributions, Stat. Probab. Lett., 80(17-18):1265-1270, 2010. 